\title{
Nonlocal resources in the presence of Superselection Rules
}

\author{
N. Schuch, F. Verstraete, and J.I. Cirac \\ Max-Planck-Institut für Quantenoptik, Hans-Kopfermann-Str. 1, Garching, D-85748, Germany.
}

\begin{abstract}
Superselection rules severely alter the possible operations that can be implemented on a distributed quantum system. Whereas the restriction to local operations imposed by a bipartite setting gives rise to the notion of entanglement as a nonlocal resource, the superselection rule associated with particle number conservation leads to a new resource, the superselection induced variance of local particle number. We show that, in the case of pure quantum states, one can quantify the nonlocal properties by only two additive measures, and that all states with the same measures can be asymptotically interconverted into each other by local operations and classical communication. Furthermore we discuss how superselection rules affect the concepts of majorization, teleportation and mixed state entanglement.
\end{abstract}

PACS numbers: 03.67.-a,03.65.Ud,03.67.Mn

One of the most remarkable and deepest discoveries in the field of quantum information theory has been the fact that the amount of nonlocality (i.e. entanglement) of pure bipartite quantum states can be quantified using a single number, namely the entropy of entanglement (EoE) [1]. This is due to the fact that all pure states with the same amount of EoE can be asymptotically interconverted into each other by local operations and classical communication (LOCC) and thus into singlets. The modern look at entanglement is to see it as a resource that allows to do tasks that are otherwise impossible, e.g. teleportation [2], dense coding [3] and quantum computing [4], and hence the EoE quantifies the nonlocal resources associated to a given state.

The whole field of entanglement theory can be formally seen as the study of the possible conversions of multipartite states given the natural restriction that one can only implement local operations and communicate by a classical channel (LOCC). It is evident that the very presence of a constraint gives rise to the concept of a resource that enables one to overcome this constraint: the essence of e.g. teleportation is the fact that the presence of entanglement allows to implement global quantum operations by local means, and hence to overcome locality constraints. Additional natural constraints should therefore give rise to new resources and to new interesting physics and applications.

It was noted by Popescu [5] that such an additional restriction applies in many physical systems in the form of a superselection rule (SSR) [6]. In [7], we considered the constraint of particle number conservation, which was motivated by current quantum optical experiments on e.g. cold atomic gases [8]. We indeed observed that the extra limitation leads to new applications that are impossible without SSR: perfect data hiding [9] becomes possible in the presence of SSR [7]. On the other hand, it was shown in [7, 10 that the extra resource of a common reference frame, in the form of a nonlocal state, allows to overcome the restrictions imposed by the SSR (note that [10] also addresses nonabelian SSR). The cen- tral contribution of this paper is to quantify this new resource induced by SSR. Fortunately, it turns out that there is a unique way of achieving this, and this measure will be called superselection induced variance $(\mathrm{SiV})$. Of course the quantification of entanglement has also to be reconsidered in the presence of SSR (see e.g. [7, 11, 12]), but we will see that the concept of EoE does not have to be altered. Furthermore, we will show that the nonlocal properties of pure bipartite quantum states subject to SSR are completely captured by specifying two (additive) measures, namely the entropy of entanglement (EoE) and the superselection induced variance $(\mathrm{SiV})$. This follows from the fact that, asymptotically, the Hilbert space decouples into two complementary Hilbert spaces, each one associated to one resource. In analogy with teleportation, the additional resource allows to implement global quantum operations locally [7], although not with unit fidelity; this defect follows from the fact that the Hilbert space associated to the $\mathrm{SiV}$ has a direct sum structure as opposed to a tensor product structure.

Let us first define the SSR under consideration more precisely. Following 7], we consider a set of particles and the corresponding Hilbert space $H$. We can always decompose $H=\bigoplus_{N=0}^{\infty} H_{N}$ where $H_{N}$ is a subspace with a total number $N$ of particles. We assume that the particle number is a superselection observable, in the sense that the corresponding operator $\hat{N}$ commutes with all observables. This immediately imposes that pure states have only support in one superselection sector $H_{N}$. If bipartite systems are considered however, $H_{N}$ can be decomposed as $H_{N}=\bigoplus_{n=0}^{N}\left(H_{n}^{A} \otimes H_{N-n}^{B}\right)$, where $H_{n}^{A}$ $\left(H_{n}^{B}\right)$ denotes a Hilbert space corresponding to system A (B), with $n$ particles. The limitation induced by the SSR precisely consists in the fact that all local observables have to commute with the local particle number operator. Summarized, the particle number operator is given by $\hat{N}_{A B}=\hat{N}_{A} \otimes \mathbb{1}_{B}+\mathbb{1}_{A} \otimes \hat{N}_{B}$, all physical density operators have to commute with $\hat{N}_{A B}$, and all local observables with $\hat{N}_{A}$ or $\hat{N}_{B}$. Clearly, local variations of particle number can occur as long as the global particle 
number remains constant. As shown in 7], the coherence between the parts of the wavefunction with different local particle number cannot be observed locally, and this is precisely the new kind of nonlocality that arises as a consequence of SSR.

Let us start by considering the analogue of the Schmidt decomposition in the presence of SSR, parameterizing all nonlocal properties of a quantum state. It is readily verified that the usual Schmidt decomposition on a state subject to SSR yields local bases with all basis vectors having a fixed particle number. Therefore we can parameterize the Schmidt coefficients as $\left\{\lambda_{i}^{n}\right\} \equiv\left\{\vec{\lambda}^{n}\right\}$, where $n$ denotes Alice's local particle number and $i$ the $i$ 'th Schmidt coefficient in the corresponding subspace. When no SSR apply, the possible conversions of one state into another one can elegantly be described by applying the concepts of majorization [13, 14] to the Schmidt coefficients. Let us now prove that a similar theorem applies in the presence of SSR: given a state $\psi$ with Schmidt coefficients $\left\{\vec{\lambda}^{n}\right\}$, then this state can be converted into the set of states $\left\{\phi_{\alpha}\right\}$ with corresponding probabilities $\left\{p_{\alpha}\right\}$ and Schmidt coefficients $\vec{\mu}_{\alpha}^{n}$ if and only if $\forall n: \vec{\lambda}^{n} \prec \sum_{\alpha} p_{\alpha} \vec{\mu}_{\alpha}^{n}$. The necessity of the condition is obvious from the results without SSR, because all POVM elements that one can implement have to commute with the local particle number operator and are hence block-diagonal. Let us prove the sufficiency for the case where there is only one state $\phi=\phi_{1}$, and hence $p_{1}=1$. From [13], it follows that for each subspace of constant local particle number, there exists an appropriate POVM with elements $\left\{M_{i}^{n}\right\}$, each producing the desired state with probability $p_{i}^{n}$. The complete conversion can therefore be obtained by taking a direct sum of the corresponding operators: $\bar{M}_{i}=\bigoplus_{n} M_{i}^{n}$. The problem however is that it will typically not hold that $p_{i}^{n}=p_{i}^{m}, n \neq m$, but this defect can readily be cured by subdividing each element $M_{i}^{n}$ into copies of itself but with different weights such that $\forall n, m: p_{i}^{n}=p_{i}^{m}$, which ends the proof. The more general proof follows immediately.

Let us next try to formulate an asymptotic version of the previous theorem. We know that, in the case of an asymptotic amount of copies of a given state and no SSR, the majorization criterion converges to the entropic criterion 1]. Analogously, consider the probability distribution associated to the variation in local particle number $\left(p_{n}=\sum_{i} \lambda_{i}^{n}\right)$. In the case of an asymptotic amount of copies, the central limit theorem dictates that this distribution becomes Gaussian and can completely be characterized by its mean and variance. Let us therefore define the measure superselection induced variance $(\mathrm{SiV})$ for a pure bipartite state as the variance in the local particle number:

$$
V(\psi)=4\left(\left\langle\psi\left|\hat{N}_{A}^{2} \otimes \mathbb{1}_{B}\right| \psi\right\rangle-\left\langle\psi\left|\hat{N}_{A} \otimes \mathbb{1}_{B}\right| \psi\right\rangle^{2}\right)
$$

The factor was included to normalize the $\mathrm{SiV}$ such that $V(|01\rangle+|10\rangle)=1$. Note that the $\mathrm{SiV}$ is additive $[V(\psi \otimes$
$\phi)=V(\psi)+V(\phi)]$ and symmetric under interchange of $A$ and $B$. It can also readily be proven that the $\mathrm{SiV}$ is an entanglement monotone [15], since after any local physical POVM measurement $\left\{M_{i}\right\}$, it holds that the expected $\mathrm{SiV}$ decreases:

$$
\sum_{i}\left\langle\psi\left|M_{i}^{\dagger} N_{A}^{2} M_{i}\right| \psi\right\rangle-\sum_{i} \frac{\left\langle\psi\left|M_{i}^{\dagger} N_{A} M_{i}\right| \psi\right\rangle^{2}}{\left\langle\psi\left|M_{i}^{\dagger} M_{i}\right| \psi\right\rangle} \leq \frac{1}{4} V(\psi)
$$

The inequality follows by using Cauchy-Schwarz and the properties of the POVM-elements $\left(\left[M_{i}, N_{A}\right]=\right.$ $\left.0, \sum_{i} M_{i}^{\dagger} M_{i}=\mathbb{1}\right)$. The $\mathrm{SiV}$ therefore fulfills all requirements for a good entanglement measure, and we expect it to completely characterize the particle number variation in the asymptotic limit. This can be formalized in the following central result of this paper:

Theorem 1 Consider $N$ copies of a state $|\phi\rangle$ with entropy of entanglement $E(\phi)$ and SiV $V(\phi)$, then there exists an asymptotically reversible conversion

$|\phi\rangle^{N} \leftrightarrow\left[|01\rangle_{A}|10\rangle_{B}+|10\rangle_{A}|01\rangle_{B}\right]^{\otimes N E(\phi)} \otimes \sum_{n} c_{n}|n\rangle_{A}|N-n\rangle_{B}$,

where the coefficients $c_{n}$ are Gaussian distributed with SiV NV $(\phi)$, and $|n\rangle$ denotes the state $|\underbrace{1 \cdots 1}_{n} 0 \cdots 0\rangle$.

Proof: In order to reduce the notational overhead, we will outline the proof for a state $|\phi\rangle=\sqrt{p_{0}}|0\rangle_{A}|1\rangle_{B}+$ $\sqrt{p_{1}}|1\rangle_{A}|0\rangle_{B}$. Given $N$ copies of the state $|\phi\rangle$, it holds that

$$
\begin{aligned}
|\phi\rangle^{\otimes N} & =\sum_{n} \sqrt{c_{n}}\left|\psi_{N-n, n}\right\rangle \quad ; \quad c_{n}=p_{0}^{n} p_{1}^{N-n}\left(\begin{array}{c}
N \\
n
\end{array}\right) \\
\left|\psi_{N-n, n}\right\rangle & \propto \sum_{\substack{i_{1} i_{2} \cdots i_{N}=0 \\
\sum_{j} i_{j}=N-n}}^{1}\left|i_{1} \cdots i_{N}\right\rangle_{A}\left|1-i_{1} \cdots 1-i_{N}\right\rangle_{B}
\end{aligned}
$$

We have decomposed the state into components with constant local particle number $\left|\psi_{N-n, n}\right\rangle$, and each of these is a maximally entangled state with Schmidt number $\left(\begin{array}{l}N \\ n\end{array}\right)$. In the limit of large $N$, the distribution of $c_{n}$ approaches a Gaussian with width $\propto \sqrt{N}$, and hence we can restrict the sum over $n$ to the $\epsilon$-typical subspaces $\mathcal{S}$ for which $\left(\begin{array}{l}N \\ n\end{array}\right) \geq 2^{N\left[H\left(p_{0}\right)-K \epsilon\right]}[16]$. Here $H(p)$ denotes the Shannon entropy of the distribution $(p, 1-p)$. Using the previously derived majorization results, there exists an LOCC protocol that can (coherently) decrease the Schmidt rank in all $\left|\psi_{N-n, n}\right\rangle$ to the value $2^{N\left(H\left(p_{0}\right)-K \epsilon\right)}$ without changing their relative weight. By an appropriate transformation of the local basis states, and by adding local ancilla states to get the correct average particle number, this new state can be rewritten as

$$
\left[|01\rangle_{A}|10\rangle_{B}+|10\rangle_{A}|01\rangle_{B}\right]^{\otimes N[H(p)-K \epsilon]} \otimes \sum_{n \in \mathcal{S}} c_{n}|N-n\rangle_{A}|n\rangle_{B}
$$




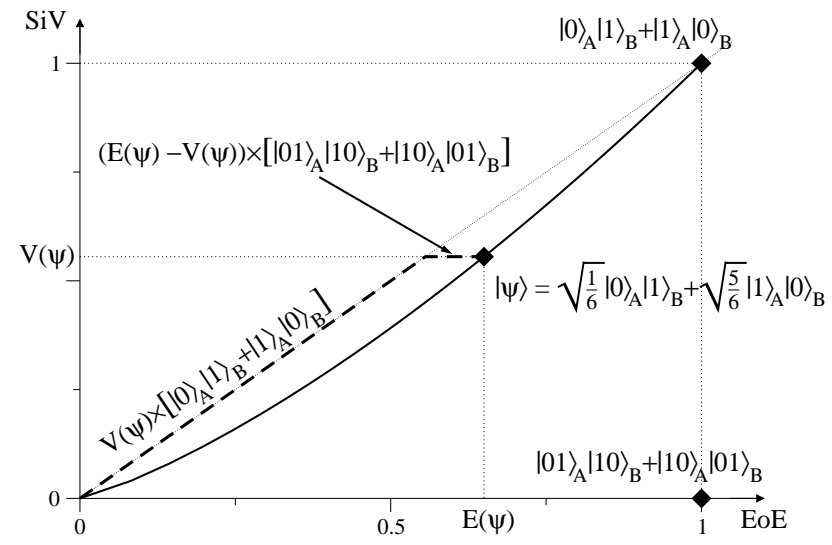

FIG. 1: Diagram representing the two nonlocal resources associated to a state $|\psi\rangle=\sqrt{1 / 6}|01\rangle+\sqrt{5 / 6}|10\rangle$.

where $c_{n}$ is a Gaussian distribution with variance $N p(1-$ $p)=N V(\phi) / 4$. Note that the sum can again be extended to all $n$ with arbitrary fidelity, and this completes the distillation direction of the proof.

To prove the dilution step, it is enough to observe that, starting from the state

$$
\left[|01\rangle_{A}|10\rangle_{B}+|10\rangle_{A}|01\rangle_{B}\right]^{\otimes N[H(p)+K \epsilon]} \otimes \sum_{n \in \mathcal{S}} c_{n}|N-n\rangle_{A}|n\rangle_{B}
$$

(note the term $+K \epsilon$ ), the majorization step can be reversed and hence the $\epsilon$-typical subspaces can be recovered. This completes the proof for the case of qubits. The proof for the general case is completely analogous and will be presented elsewhere 17].

It follows that a state can be interconverted into a tensor product of a state with only EoE and no $\mathrm{SiV}$, and another part that contains a negligible amount of EoE but all SiV. Indeed, the state $|01\rangle_{A}|01\rangle_{B}+|10\rangle_{A}|10\rangle_{B}$ has constant local particle number and therefore SSR impose no restrictions whatsoever on the local operations that can be applied on this part (see 7]). On the other hand, the part with Gaussian distributed coefficients contains all $\mathrm{SiV}$ and only a logarithmic amount of entanglement $(\simeq \log (N))$, therefore solely containing the $\mathrm{SiV}$ resources of the original state $|\phi\rangle$. Note however that in the case of qubits, it is readily verified that the EoE always exceeds the $\mathrm{SiV}$, and an equivalent version of the previous theorem would be:

$$
\begin{array}{r}
|\psi\rangle^{\otimes N} \leftrightarrow\left[|01\rangle_{A}|10\rangle_{B}+|10\rangle_{A}|01\rangle_{B}\right]^{\otimes N[E(\psi)-V(\psi)]} \\
\otimes\left[|0\rangle_{A}|1\rangle_{B}+|1\rangle_{A}|0\rangle_{B}\right]^{\otimes N V(\psi)}
\end{array}
$$

As an example, this decomposition has been illustrated in Figure 1 for the state $|\psi\rangle=\sqrt{1 / 6}|01\rangle+\sqrt{5 / 6}|10\rangle$.

The important insight of the given theorem is that, asymptotically and in the presence of SSR, the Hilbert space decouples into a tensor product of a Hilbert space equipped with a tensor product structure and another one equipped with a direct sum structure, the first one related to the $\mathrm{EoE}$ and the second one to the $\mathrm{SiV}: H_{d}^{\otimes n} \simeq$ $H_{d}^{\otimes n-\log _{d} n} \otimes\left[\oplus_{n} H_{d-1}\right]$. The resource of EoE is very powerful and well understood by now due to the concept of teleportation [2]: it allows to completely overcome the constraints imposed by LOCC as it allows to implement global operations locally. As shown in [7], a similar result holds for the SiV. Indeed, in the presence of SSR, it is impossible to distinguish the Bell states $\left|\phi^{ \pm}\right\rangle=|01\rangle \pm|10\rangle$ by LOCC, which is the basis for a perfect data hiding protocol; however, an extra state $|01\rangle+|10\rangle$ allows to obtain (partial) information and hence to the $\mathrm{SiV}$ can be used as a resource to overcome the limitations induced by the SSR.

The crucial difference between the two resources however is their associated Hilbert space structure: the dimension of the respective Hilbert spaces grows exponential versus linear in the number of qubits. A direct consequence is that e.g. the number of bits that can be hidden securely [7] in $N$ qubits scales only as $\log _{2}(N)$. More fundamentally, we will show that this implies that the resources of $\mathrm{SiV}$ needed to completely overcome the locality constraints scale superlinearly in the number of nonlocal particles under consideration: teleportation of $N \rightarrow \infty$ particles is only possible using an amount of maximally entangled particles in the order of $N^{1+\epsilon}$, such that the quotient of the Hilbert space of the resources needed with that one of the state to be teleported diverges. As already noticed in 7], this implies that in the presence of SSR, having a bounded amount of entanglement and a classical channel is not equivalent anymore to having a quantum channel: in the presence of SSR, it is only possible to teleport one qubit with unit fidelity in the limit of an unbounded amount of SiV.

Indeed, consider a resource state $|\psi\rangle \propto \sum_{i=0}^{M}|i\rangle_{\bar{A}} \mid M-$ $i\rangle_{B}$ which we would like to use to teleport Alice's part of a state $|\phi\rangle=\sum_{j=0}^{N} \alpha_{j}|j\rangle_{A}|N-j\rangle_{C}$ with $N$ particles shared between Alice and Charlie. Assuming $M \geq N$ and defining $\bar{n}=\min (n, N) ; \underline{n}=\max (0, n-M)$, the total state is given by

$$
\sum_{n=0}^{N+M} \sum_{j=\underline{n}}^{\bar{n}} \alpha_{j}|j, n-j\rangle_{A \bar{A}}|M-n+j\rangle_{B}|N-j\rangle_{C} .
$$

Of course Alice can only act on the subspaces with constant particle number $n$, and the best thing she can do is a Bell-measurement in the local basis $\left|\chi_{k}^{(n)}\right\rangle=$ $1 / \sqrt{\bar{n}-\underline{n}+1} \sum_{l=\underline{n}}^{\bar{n}} e^{i 2 \pi l k /(\bar{n}-\underline{n}+1)}|l, n-l\rangle$ and communicate the result to Bob who applies a unitary operation conditioned on her result. Perfect teleportation has taken place iff $\underline{n}=0$ and $\bar{n}=N$, and the probability that this occurred is exactly given by $1-N /(M+1)$. It follows that perfect teleportation is only possible when $M$ scales superlinearly with $N$, proving the previously stated result. 
We conclude that both EoE and $\mathrm{SiV}$ represent resources that allow for teleportation, although the qualitative behavior is different.

The fact that $\mathrm{SiV}$ represents a resource independent of EoE can also be appreciated by looking at the case of mixed states. As shown in 7], there exist mixed states that are not entangled in the usual sense but cannot be prepared locally due to the SSR-constraints. In the light of $\mathrm{SiV}$, this means that they are nonlocal because $\mathrm{SiV}$ is needed to create them (but no EoE), and it was furthermore shown that these mixed states can be used to do things that are otherwise impossible. The present results allow to turn these arguments into quantitative ones.

Therefore, it is natural to define the entanglement and the variance of formation as

$$
\begin{aligned}
E_{F}^{\mathrm{SSR}}(\rho) & =\min _{\left\{i, \psi_{i}\right\}} \sum_{i} p_{i} E\left(\psi_{i}\right) \\
V_{F}^{\mathrm{SSR}}(\rho) & =\min _{\left\{p_{i}, \psi_{i}\right\}} \sum_{i} p_{i} V\left(\psi_{i}\right)
\end{aligned}
$$

where the ensemble $\left\{\psi_{i}\right\}$ has to be conform to the SSR. The entanglement cost and variance cost 18] are then defined as the regularized versions of it $\left(E_{c}^{\mathrm{SSR}}(\rho)=\lim _{N \rightarrow \infty} E_{F}^{\mathrm{SSR}}\left(\rho^{\otimes N}\right) / N ; V_{c}^{\mathrm{SSR}}(\rho)=\right.$ $\left.\lim _{N \rightarrow \infty} V_{F}^{\mathrm{SSR}}\left(\rho^{\otimes N}\right) / N\right)$. As an example, consider the state

$$
\rho=\frac{1}{4}(|00\rangle\langle 00|+| 11\rangle\langle 11|+(|01\rangle+|10\rangle)(\langle 01|+\langle 10|)) .
$$

This state is separable in the usual sense, but $E_{F}(\rho)=$ $1 / 2=V_{F}(\rho)$. To calculate its entanglement cost, we need the following result: given a density operator that is separable when no SSR apply, then its entanglement cost in the presence of SSR is zero. This can readily be proven as follows: suppose $\rho^{\otimes N}=\sum_{i} p_{i}\left|\chi_{i}\right\rangle\left|\psi_{i}\right\rangle\left\langle\psi_{i}\right|\left\langle\chi_{i}\right|$ where the $\psi_{i}, \chi_{i}$ do not obey SSR. It holds that $\rho^{\otimes N}=$ $\sum_{n} P_{n} \rho^{\otimes N} P_{n}$ where $P_{n}$ is the global projector on the (global) subspace of $n$ particles. Acting with $P_{n}$ on a separable state, at most $\log _{2}(N)$ EoE can be created, and therefore the entanglement cost will behave as $\log _{2}(N) / N$, concluding the proof. On the other hand, the variance cost for the particular state $\rho$ can easily be shown to be additive, as the subspaces of constant local particle number are pure 17].

Analogously to the normal case of mixed states, one can readily define quantities like entanglement of distillation [19] and variance of distillation. The recurrence schemes of [20] can be adapted to the SSR-case, and it can be shown that any qubit state with a vanishing $E_{c}^{\mathrm{SSR}}$ but nonvanishing $V_{F}^{\mathrm{SSR}}$ can be converted into any other one of this class, and any state with a non-vanishing $E_{c}^{\mathrm{SSR}}$ can be purified to a perfect singlet $|01\rangle+|10\rangle$. A more elaborate exposition of these results will be presented elsewhere [17.

In conclusion, we have developed the theory of entanglement for bipartite quantum states subject to SSR. We made the obvious observation that, both in the case of LOCC and SSR, there exist resources that let you overcome the constraints. We identified two independent resources that asymptotically completely characterize the amount of nonlocality present in a pure state, and showed that these lead to the necessary and sufficient condition for interconvertibility of quantum states under LOCC. One resource is characterized by the familiar entropy of entanglement, while the other one by the superselection induced variance. We discussed how the resources allow to overcome the LOCC- respectively the SSR-constraints by teleportation, although the $\mathrm{SiV}$ is fundamentally different from the EoE because of the direct sum structure of its associated Hilbert space. We concluded this paper by stating some intriguing properties for the entanglement properties of mixed bipartite states subject to SSR.

This work was supported in part by the E.C. (RESQ QUIPRODIS) and the Kompetenznetzwerk "Quanteninformationsverarbeitung" der Bayerischen Staatsregierung.

[1] C.H. Bennett, H.J. Bernstein, S. Popescu, and B. Schumacher. Phys. Rev. A, 53:2046, 1996.

[2] C.H. Bennett et al. Phys. Rev. Lett., 70:1895, 1993.

[3] C.H. Bennett and S.Wiesner. Phys. Rev. Lett., 69:2881, 1992.

[4] P.W. Shor. SIAM J.Sci.Statist.Comput., 26:1484, 1997.

[5] S. Popescu, personal communication.

[6] G.C. Wick, A.S. Wightman and E.P. Wigner, Phys. Rev. $88: 101,1952$.

[7] F. Verstraete and J.I. Cirac. Phys. Rev. Lett., 91:010404, 2003.

[8] E.A. Cornell and C.E. Wieman, Rev. Mod. Phys. 74:875, 2002; W. Ketterle, Rev. Mod. Phys. 74:1131, 2002.

[9] B.M. Terhal, D.P. DiVincenzo, and D.W. Leung. Phys. Rev. Lett., 86:5807, 2001.

[10] A. Kitaev, D. Mayers and J. Preskill. quant-ph/0310088

[11] T. Rudolph and B.C. Sanders. Phys. Rev. Lett., 87:077903 (2001).

[12] H.M. Wiseman and J.A. Vaccaro. Phys. Rev. Lett., 91:097902, 2003; S.D. Bartlett and H.M. Wiseman. Phys. Rev. Lett., 91:097903, 2003; H.M. Wiseman et al. quant-ph/0309046

[13] M.A. Nielsen. Phys. Rev. Lett., 83:436, 1999.

[14] D. Jonathan and M. Plenio. Phys. Rev. Lett., 83:1455, 1999.

[15] G. Vidal. Jour. of Modern Optics, 47(2/3):355, 2000.

[16] T.M. Cover and J.A. Thomas. Elements of Information Theory. Wiley-Interscience, 1991.

[17] N. Schuch, F. Verstraete and J.I. Cirac, in preparation.

[18] P. Hayden, M. Horodecki and B.M. Terhal. J. Phys. A, 34:6891, 2001.

[19] C.H. Bennett et al. Phys. Rev. Lett., 76: 722, 1996.

[20] C.H. Bennett, D.P. DiVincenzo, J.A. Smolin, and W.K. Wootters. Phys. Rev. A, 54:3824, 1996. 